\title{
FACTORES SOCIO ECONÓMICOS Y DE SERVICIOS DE SALUD ASOCIADOS CON LA MORTALIDAD MATERNA: UNA REVISIÓN
}

\author{
SOCIO ECONOMIC AND HEALTH SERVICES RELATED TO \\ MATERNAL MORTALITY: A REVIEW
}

\author{
Cordero Rizo Marcia Zulema ${ }^{1}$ \\ González Guillermo Julián²
}

Correspondencia: corderorizo@hotmail.com

Recibido para evaluación: enero - 06 - 2011. Aceptado para publicación: marzo - 14- 2011

\section{RESUMEN}

Introducción: aproximadamente 15.000 mujeres mueren cada año en América Latina y el Caribe por causas relacionadas con el embarazo. La tasa derivada de la mortalidad materna para la región, es de 130 muertes maternas por 100.000 nacidos vivos, cifra más elevada que la observada en los países desarrollados.

Objetivo: identificar factores socio económicos y la prestación de los servicios de salud y su impacto en la mortalidad materna.

Metodología: se realizó una búsqueda de la literatura en bases de datos electrónicas mediante palabras clave con relación al tema, para detectar los factores socioeconómicos y la prestación de los servicios de salud. Se buscaron artículos desde enero de 1998 hasta diciembre del 2010. Se identificaron 16 factores (o temas) los cuales se observaron para establecer relación con mortalidad materna.

Resultados: los temas o factores socioeconómicos asociados a mortalidad materna identificados fueron el nivel de educación, paridad materna, la edad, región, estado civil, condiciones de la vivienda y de los servicios de salud, controles prenatales y seguridad de los servicios de salud.

Conclusión: el bajo nivel socioeconómico y por ende la pobreza constituyen determinantes de muerte materna. La falta de disponibilidad y los impedimentos al acceso a los cuidados prenatales también tienen una influencia significativa. Rev. cienc.biomed. 2011; 2(1): 77-85

\section{PALABRAS CLAVES}

Factores socioeconómicos. Mortalidad materna. Organización médica. Cuidados de salud. Servicios de salud.

\section{SUMMARY}

Background: approximately 15.000 women die each year in Latin America and the Caribbean for pregnancy-related causes. The resulting rate of maternal mortality for the region is 130 maternal deaths per 100,000 live births, higher than that observed in developed countries.

Objective: to identify socio-economic factors and the provision of health services and their impact on maternal mortality.

Methods: we performed a literature search in electronic databases using key words relating to the subject, to detect socioeconomic factors and the provision of health

\footnotetext{
1 Enfermera con Magister en Salud sexual y Reproductiva. Estudiante de doctorado en Ciencias de la Salud Pública. universidad de Guadalajara, Jalisco - México.

2 Dr. en Ciencias de la Salud; Profesor - Investigador Titular C, CUCS, Universidad de Guadalajara. México.
} 
services. Articles were searched from January 1998 through December 2010. We identified 16 factors (or themes) which were observed to establish relationships with maternal mortality.

Results: subjects or socioeconomic factors associated with maternal mortality identified were education, maternal parity, age, region, marital status, housing conditions, health services, prenatal care and safety of health services.

Conclusion: low socioeconomic status and therefore poverty are determinants of maternal death. The lack of availability and restrictions on access to prenatal care also has significant influence. Rev.cienc.biomed. 2011; 2(1): 77-85

\section{KEYWORD}

Socioeconomic factors. Maternal mortality. Health care. Health services. Medical organization.

\section{INTRODUCCIÓN}

Según la Organización Mundial de la Salud (1), en todo el mundo murieron 493.000 mujeres en el año 1998 por complicaciones del embarazo y el parto. De estas muertes, el $99 \%$ ocurrieron en países en desarrollo, donde la mortalidad materna es 18 veces mayor que en los países desarrollados (1). Además, las Naciones Unidas estiman que 536, 000 mujeres mueren cada año a partir de complicaciones durante el embarazo y parto. Aproximadamente 22.000 muertes maternas se producen anualmente en la región de América Latina y el Caribe. Más de medio millón de mujeres siguen muriendo cada año durante el embarazo o el parto (2), lo que representa un problema de salud pública para la comunidad internacional.

En Nicaragua, el segundo país más pobre de América Latina y el Caribe, la relación de mortalidad materna ha llegado tan alto como 230 muertes maternas por cada 100.000 nacidos vivos en los últimos años (1).

Ante el panorama del fenómeno de la mortalidad materna, el quinto Objetivo de Desarrollo del Milenio de la ONU tiene como objetivo mejorar la salud materna y reducir la mortalidad materna (MMR) relacionada en tres cuartas partes entre 1990 y 2015.

La mortalidad materna representa una serie de complicaciones médicas en todas las sociedades. En muchos países de bajos ingresos, las complicaciones durante el embarazo y en niños son la principal causa de muerte alrededor de mujeres en edad reproductiva. La gran mayoría de las muertes maternas así como problemas asociados se pueden prevenir a través de servicios apropiados de salud reproductiva antes, durante y después del embarazo y por medio de intervenciones para salvar vidas si surgieran complicaciones (2).

Muchos factores de riesgo han sido asociados con mortalidad materna. Estos incluyen enfermedades, factores de servicios de salud, factores reproductivos y factores socioeconómicos. Generalmente la edad de la madre, la paridad, el estado de salud antes del embarazo y el estado nutricional se relacionan con mortalidad materna (4). Además, se han encontrado asociaciones entre la mortalidad materna, el nivel de educación y el acceso a la atención de la salud (1).

La falta de disposición de servicios de salud materna en países en desarrollo, como resultado de condiciones sociales y económicas, contribuye de manera significativa a mortalidad materna. La atención prenatal y planificación familiar muestran ser indispensables para la prevención de las muertes maternas. En este sentido, el acceso a la atención primaria en salud puede ayudar a las embarazadas y su comportamiento con respecto a la utilización de los servicios de salud modernos (5).

Debido al impacto social y la relevancia de comprender el fenómeno de la mortalidad materna, el objetivo de este trabajo fue describir la información disponible en publicaciones sobre los factores socioeconómicos y lo referente a servicios de salud asociados a la mortalidad materna. 


\section{METODOLOGÍA}

En varias bases se realizó búsqueda de datos que abarcaron los factores socio económicos y de servicios de Salud asociados a Mortalidad Materna: EBESCO, ISI Web of Knowledge, Pub Med, Scielo, fueron consultadas. Las palabras claves incluidas fueron: Factors, Socioeconomic, Associated, Maternal, Mortality, Care, Health, Medical, Organization.

Datos duplicados en las bases de datos fueron excluidos. Se evaluaron los artículos sobre mortalidad materna publicados entre 1998 y 2010 . Para evaluar las características de trabajo establecido sobre mortalidad materna se llevó a cabo una revisión identificados a partir de las bases de datos mencionadas anteriormente los términos de búsqueda: "la mortalidad materna." Los criterios de inclusión empleados para la selección de estudios fueron: ser estudios empíricos (cualitativos y cuantitativos), que estudiaran los factores socio económicos y

\section{FLUJOGRAMA DE SELECCIÓN Pub Med, Scielo, ISI Web of Knowledge, Redalyc, Ebesco.}

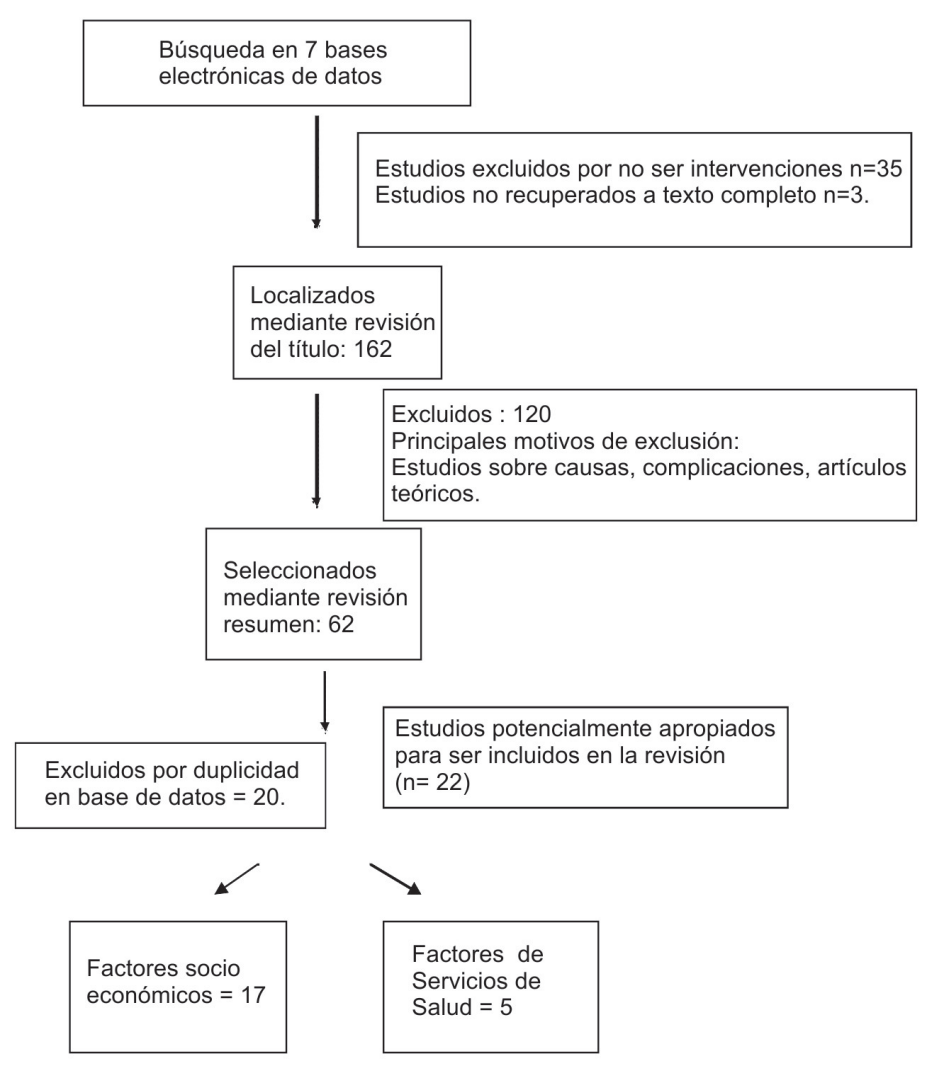

de servicios de salud asociados a mortalidad materna, publicados en inglés y español. Se excluyeron artículos que trataban de las causas de mortalidad materna y de aspectos clínicos como complicaciones durante el embarazo, parto y puerperio y mortalidad Infantil. Después de revisar 162 artículos, se seleccionaron los que exclusivamente trataban sobre factores socio económicos y de servicios de salud asociados a mortalidad materna. El proceso de selección se señala en la Figura No. 1.

Los artículos se seleccionaron según su relevancia para los temas. Asimismo, no fueron removidos de la revisión por presentar baja calidad, aunque algunos pudieran tener problemas de diseño y reporte de la información. Para su análisis, se realizaron matrices para cada tema con la finalidad de describir los hallazgos, dirección y magnitud de las asociaciones, fortaleza de la calidad, contexto, características de los participantes y el tipo y diseño de los estudios.

La mayoría de los estudios incluidos en la revisión son cuantitativos. Las regiones dominantes en las que se realizaron los estudios son Europa y América del Norte (representado por EUA). La localidad de los sujetos generalmente es rural, se estudia en mujeres y principalmente se realizan en el entorno de atención, hospitalización y cuidados obstétricos.

\section{FACTORES SOCIOECONÓMICOS}

\section{A. Educación}

Evaluando mortalidad materna en un país de África, Konteh 1990 (7), revela una alta tasa de analfabetismo, ya que la mayoría de las mujeres nunca habían asistido a alguna institución educativa. Un número considerable de mujeres tenían solo educación primaria y la proporción de mujeres que iban a la escuela disminuyó con la residencia en zonas distantes. Muchos estudios sostienen que la utilización de servicios médicos se incrementa con el nivel de educación. Sin embargo, se necesitan más estudios para demostrar si existe una asociación 
entre la mortalidad materna y educación de la mujer, sin embargo el nivel de educación no fue considerado como factor de riesgo en el análisis multivariado (8). La educación materna estuvo negativamente asociada con la mortalidad materna. El promedio de odds ratio de mortalidad materna de mujeres con educación secundaria comparado por encima de aquellas cuya educación es primaria o educación no formal es de 0.56; el autor no menciona intervalos de confianza (5). En otro estudio se señala que la falta de educación formal básica es un factor asociado con las tasas de mortalidad materna. Uno de los principales factores que se ha documentado es la educación materna donde los hallazgos ya mencionados muestran variabilidad en sus resultados, sin embargo, en la presente revisión no se ha encontrado evidencia solida que sugiera que la mortalidad materna esté directamente relacionada con la educación de la mujer (9).

\section{B. Religión}

El factor religioso afecta a la mortalidad materna en larga escala. La religión es un problema no solamente por los efectos de posición social de la mujer sino por las creencias y tradiciones perjudiciales en relación al parto, por ejemplo la costumbre islámica de Purdah trata de la reclusión de las mujeres de la vista de los hombres. Purdah se aplica generalmente a las mujeres casadas y las niñas que han alcanzado la pubertad, aunque la práctica varía de país a país y de región a región. La práctica limita a las mujeres de sus derechos a la libertad de circulación y de asociación, de sus derechos de acceso a la educación y a otros servicios sociales (10).

\section{Paridad}

La asociación entre paridad y mortalidad materna tiene una alta significancia sobre todo en las mujeres que tienen más de cinco hijos (5). Romero et al. (4) en estudio realizado en México, asevera que en 110 casos de muertes maternas estudiadas, la media de la paridad fue de 3.6 y de los 440 controles la media fue 2.4 para una probabilidad de 0.001 . A su vez L Hoj, et al. (2002) (11), en estudio de casos y controles realizado en áreas rurales de Guinea Bissau de las 85 muertes maternas estudiadas el $29 \%$ de los casos y los controles respectivamente tenían más de 6 hijos. La multiparidad en mujeres de cualquier edad representa más peligro que el segundo o tercer nacimiento. En Jamaica, las mujeres en su quinto a noveno embarazo, tienen $43 \%$ más probabilidades de morir que las mujeres en su segundo embarazo. Los primeros nacimientos de riesgo deben ser manejados con mayor cuidado, pero se deben formular recomendaciones en cuanto a demorar el primer embarazo hasta algunos años después de la adolescencia. Los embarazos de mujeres de más edad tienden a ser de alta paridad. Los servicios de planificación familiar dirigidos a este grupo etáreo pueden llevar una reducción significativamente importante de la mortalidad materna. Existe asociación entre la multiplaridad y el riesgo de muerte materna. Parte de la problemática no mencionada en esta sesión son los hijos huérfanos nacidos de la madre que fallece situación que abona a la pobreza de cada país. El periodo intergenésico es una connotación importante. Un intervalo entre embarazos de menos de seis meses y más de cincuenta y nueve meses, está asociado con un mayor riesgo de resultados maternos adversos (17).

\section{Edad materna}

Konteh (1990) (7) establece una relación entre edad materna y paridad; describe en su estudio que alrededor del $85 \%$ de las mujeres de 45 a 49 años habían tenido seis hijos o más. El riesgo de mortalidad materna para las mujeres de 20 a 24 años suele ser menor. Las probabilidades de muerte materna parecen aumentar de manera significativa con la edad después de 25 años de edad. El mayor riesgo de mortalidad materna en relación con la edad materna elevada, se asocia con la maternidad tardía. La probabilidad media de la mortalidad materna para las mujeres mayores de 35 años es mayor que para las mujeres en los 20 - 24 años por un factor de 3.7, después de controlar los efectos de la asistencia prenatal, el nivel de la educación y la variación aleatoria entre los hospitales (5). En el estudio de Romero et al. (2007) (4) la edad media de las muertes maternas fue de 28.6 y en los controles 25.5 años. Para un odds ratio de 1.1 con un intervalo de confianza de $95 \%$ y una probabilidad de 0.001 . 
A su vez en una evaluación Lindsey (2008) (12) señala que la media de edad de mujeres que presentaron muerte materna fue de 26 años. La asociación entre la edad, estado civil y paridad, son conocidos predictores de muerte materna (13). El embarazo a edad muy temprana es un riesgo adicional de parto en todo el mundo. Entre los Países en desarrollo, Bangladesh exhibe la incidencia más alta de matrimonio precoz, $90 \%$ de las mujeres se casan antes de cumplir 18 años. Después de los 30 a 35 años de edad el riesgo de mortalidad materna también se incrementa. Este riesgo es especialmente importante en los países en desarrollo, donde las mujeres mayores tienen con frecuencia numerosos hijos. En conjunto la edad y la paridad, son factores de riesgo usados frecuentemente para definir el alto riesgo materno. Además la edad y la paridad, están consideradas determinantes de pobreza e importantes en cuanto a mortalidad materna y puede ser atribuida a condiciones desfavorables en los servicios de salud (14).

\section{E. Estado Civil}

La importancia del matrimonio y el estado civil como factores que influyen en el tamaño y la estructura del hogar y la familia es patente y ha sido documentada ampliamente por sociólogos y especialistas en demografía. Ello ocurre especialmente porque el matrimonio constituye el primer paso en la formación de la familia biológica y la mayoría de las parejas recién casadas establecen su propio hogar inmediatamente después de la boda. Konteh (1990) (7) en un estudio realizado en Sierra Leona encuentra que el $40 \%$ de las mujeres están casadas a los 15 años de edad, existen pocos casos de divorcio y separación. Romero et al. (2007) (4) no encuentran significancia estadística para el estado civil.

\section{F. Pobreza}

La pobreza es un factor de alto riesgo claramente determinado en la mortalidad materna. Las mujeres de niveles socioeconómicos bajos tienen menor probabilidad que las de estratos altos de gozar de buena salud. Las primeras tienen menos oportunidades de educación formal, de buscar y recibir atención médica. El análisis de los determinantes de la pobreza requiere la profundización del conocimiento de los factores de género, las variables de vinculación sectorial, el capital humano, la dependencia económica y la demografía. Estos son algunos de los indicadores que explican la vulnerabilidad de ciertos sectores sociales exponiéndolos a mayores situaciones de riesgo o profundizar su pobreza. En las familias pobres, en las que hay pérdida de perceptores de ingresos y en las altas tasas de dependencia demográfica, por lo general se observa cierta incapacidad de actualizar el capital humano o de pasarse a actividades productivas de mayores ganancias. La elevada maternidad en la adolescencia, no muestra cambios en la última década, lo que estaría marcando o la ausencia de políticas para incidir en ese problema o la ineficacia de las que se están instrumentando en los países de la región. En términos promedios una cuarta parte de las mujeres latinoamericanas ha tenido su primer hijo antes de los 20 años de edad. En las zonas rurales, esa situación es más preocupante, donde se eleva al $30 \%$. La tasa de fecundidad disminuye en las ciudades con relación al campo entre un $15 \%$ y $20 \%$. La impresión general es que las mujeres víctimas de muertes maternas murieren a causa de la pobreza. Estas personas usualmente no pueden pagar el costo de los medicamentos, operaciones, transporte y cuidados postoperatorios. Tampoco reciben atención prenatal y posnatal, ni el recurso para cubrir el costo de su suministro en hospitales. La mayoría de las mujeres que fallecen en gestación o por causas relacionadas con el embarazo están desnutridas y anémicas, porque carecen de una dieta adecuada y equilibrada (7). Los bajos factores socioeconómicos tales como vivir en área rural, desempleo, pobre higiene y no disponibilidad de cuidados antenatales tienen una influencia en la alta mortalidad materna en Indonesia, las mujeres con bajo estatus socioeconómico son a menudo ignoradas (8).

\section{G. Condiciones de la vivienda}

En un estudio de 85 muertes maternas, el $28 \%$ no tenía acceso al agua dentro de la vivienda y el $71 \%$ tenía que traerla de fuera. De los controles el 32\% tenían acceso al agua dentro de la vivienda y el $67 \%$ debían traerla de fuera. La disponibilidad de letrina 
entre las mujeres que presentaron muerte materna tuvo significancia en el análisis multivariado pero no con la presencia de embarazo gemelar ni la distancia al hospital. (11). Es interesante que la falta de disponibilidad de aseo facilite la aparición de riesgo de mortalidad materna. Esto implica que los beneficios de la salud podrían ser maximizados solo cuando en las viviendas tengan agua segura y servicios sanitarios (8). Tanto las condiciones generales de la vivienda en la comunidad, como la disponibilidad de casas suministradas a precios razonables y las políticas del gobierno en materia de vivienda son factores influyentes.

\section{H. Área de residencia}

J Hoj et al (2002) (11) muestran que de 85 muertes maternas estudiadas, el $15 \%$ eran de área urbana y un $84 \%$ del área rural. Ronsmans et al. (2003) (15), describen que los intervalos de confianza son amplios, pero la mortalidad materna fue significativamente mayor en las zonas rurales que en las zonas urbanas. Se observó una notable agrupación de los niveles de uso de servicios en las zonas urbanas y rurales. Análisis estratificado en zonas urbanas y rurales no reveló una tendencia clara, sin embargo, ninguna de las asociaciones dentro de los estratos fueron significativas. Este estudio revela grandes desigualdades en el acceso a la atención obstétrica y los niveles de mortalidad materna en las zonas urbanas y rurales en África del oeste. La mayoría de las mujeres rurales dan a luz en casa, en la ausencia de atención especializada, mientras que las mujeres urbanas tienden a dar a luz en un hospital con un personal calificado. Al mismo tiempo, la mortalidad materna es muy alta en las zonas rurales, y sustancialmente más bajos en las zonas urbanas. Sin embargo, dentro de las áreas urbanas o rurales, no hubo asociación evidente entre la mortalidad materna y la atención obstétrica especializada, los nacimientos en el hospital o las cesáreas.

\section{Lugar de ocurrencia de la muerte}

Bashour H, et al. (2009) (3) describen las muertes maternas según lugar del parto y lugar de ocurrencia de la muerte. Comparando la información de prevalencia de muertes maternas desglosándolos en tres grupos los hallazgos fueron: las mujeres que parieron en casa; se encontró que la muerte de la madre en casa equivale al $13.3 \%$, mujeres que murieron durante el traslado al hospital corresponden al $31 \%$ y las que murieron en el hospital al 55.6\%. En el grupo de madres que parieron en el hospital se encontró que las que murieron en casa corresponden al $1.8 \%$, murieron durante el traslado al hospital un 3.5\% y mujeres que murieron en el hospital un $94.7 \%$. Y el grupo de mujeres que parieron en clínicas privadas pero murieron en la casa un $21.4 \%$, las que murieron durante el traslado al hospital $78.6 \%$; en este grupo no hubo fallecidas en el hospital.

G Walraven (2000) (16), en otro estudio señala que el $33 \%$ de las muertes maternas ocurrieron en la casa, $33 \%$ en el Centro de Salud, $27 \%$ en el Hospital y el $5 \%$ durante el traslado. La distancia a los centros de salud y de la región de nacimiento, no son considerados factores de riesgo en la proyección oficial del programa materno infantil, pero tienen una mayor importancia más que la edad y la paridad de la mujer. La mujer tendría que dejar a su familia, a menudo durante un mes o más. Sólo un hospital en las zonas rurales de GuineaBissau ofrece instalaciones para la madre en caso de espera, así que en la mayoría de los casos la parturienta tiene que tramitar alimento y refugio. Aunque muchas mujeres no siguen los consejos que reciben, ya que es claramente poco realista, factores de riesgo deben ser evaluados cuidadosamente antes de ser ejecutado por el sistema de atención primaria.

En los países en desarrollo gran parte de las mujeres que mueren en los hospitales por causas asociadas con el embarazo llegan muy tarde para prestarles atención eficaz. Es claro que la gran mayoría de muertes maternas pueden evitarse por medio del manejo y atención tanto adecuada como oportuna, como señalan las bajas tasas de mortalidad materna de los países desarrollados.

\section{FACTORES DE SERVICIOS DE SALUD}

La intervención clínica temprana lleva a reducir los riesgos de muerte materna. Las mujeres vulnerables y con bajo nivel 
Cordero Rizo Marcia Zulema, González Guillermo Julián

socio-económico, tienen más riesgo de sufrir una enfermedad grave del embarazo y el parto, usualmente porque no tienen acceso a servicios de salud. Sin embargo, muy poco se ha hecho para evaluar los factores de riesgo de la mortalidad materna asociada con bajo nivel socioeconómico y el cumplimiento en la atención prenatal (8). Las visitas a las clínicas antenatales están ligadas o asociadas significativamente con la reducción en la mortalidad materna. Los factores que contribuyen a la mortalidad materna en Nicaragua son la falta de acceso a servicios de salud, la alta fecundidad, la violencia doméstica y las complicaciones de abortos.

Muchas estrategias se han implementado en un intento para mejorar la salud materna en todo el mundo, buscando disminuir la mortalidad materna. Programas destinados a la reducción de los tres retrasos en la búsqueda incluyen: (A) Mejorar la prevención primaria mediante la educación y los servicios.(B) Desarrollar prevención secundaria mediante la detección precoz y el tratamiento temprano de las condiciones patológicas.(C) Prevención terciaria a través del tratamiento de las condiciones para reducir la mortalidad (12).

\section{A. Control prenatal}

Es fundamental el control prenatal. $\mathrm{H}$ Bashour (2009) (3) señala que de un grupo de mujeres que tuvieron muerte materna el $77 \%$ recibió atención prenatal durante su embarazo y el $24 \%$ no recibió atención prenatal. Especial atención debe prestarse a través de la buena calidad de la atención prenatal a las mujeres con riesgo reconocido, factores tales como una enfermedad crónica o antecedentes de muerte fetal. Atención obstétrica de emergencia también necesita ser fortalecida. En otro estudio (16), de 18 muertes maternas, el I 33\% tenían de 0 a 3: Controles prenatales, $11.1 \%$ de dos a cuatro, $11 \%$ de cinco a seis controles y un $44 \%$ no sabían el número de asistencia. Las visitas prenatales tienen un efecto protector contra muerte materna. En 1994 el grupo técnico de trabajo en cuidados prenatales de la OMS recomendó un mínimo de cuatro visitas prenatales. Taguchi (8) muestra en un estudio que la media del número de visitas prenatales en mujeres que presentaron muerte materna fue de 4.6 frente al 7.1 de visitas en mujeres que no tuvieron muerte materna. Los hallazgos sugieren que las mujeres deben ser educadas acerca de la importancia del control prenatal. El profesional de salud debe estar capacitado para reconocer las complicaciones obstétricas en una fase temprana y remitir los casos de alto riesgo a centro especializados de manera oportuna (19).

\section{B. Seguridad en los servicios de salud} El cuidado de la salud en Centros de salud aumentó su sentimiento de seguridad y vigilancia, lo que contribuyó a su percepción del valor de la atención institucional y alentó a la posterior utilización de los servicios. La percepción de las mujeres sobre el uso de las instalaciones de Salud y el cuidado provee un ambiente seguro para la atención que reciben evolucionado a partir de la calidad de la atención que recibieron y sus resultados con el parto de sus embarazos anteriores. Muchas mujeres que experimentaron complicaciones con los anteriores nacimientos tuvieron un aumento del miedo y la consciencia de los riesgos al no buscar atención médica y por lo tanto buscaron atención para evitar complicaciones (12). Otros autores (20), describen la experiencia de las mujeres con el sistema de salud. Los testimonios indican que los factores estructurales y de prestación y atención médica durante los embarazos alentaron a las mujeres para la búsqueda de atención.

Las mujeres deben tener acceso ilimitado a la asistencia sanitaria, no sólo durante el parto, sino también durante toda su vida. Enfermedades Infecciosas y no infecciosas comprometen el estado de salud antes y durante el embarazo. La hepatitis, la tuberculosis, la fiebre tifoidea y la anemia fueron reportadas como factor contribuyente a la mortalidad materna. El tratamiento adecuado para estas enfermedades y todas las otras concomitantes con la gestación deben brindarse en los Centros de Salud (21). Las mujeres deben valorar los servicios prestados en las unidades de salud. El conocimiento de las mujeres y la aceptación de la importancia para el cuidado de la salud, 
embarazo saludable y las prácticas están determinados por las experiencias previas así como la comunicación formal e informal dentro de la comunidad y los hogares. Muchas mujeres que usan los servicios de salud materna creen que era importante como medio de reducir los riesgos de complicaciones y garantizar la salud de los niños. Hay una relación relativamente estrecha entre los niveles de uso de servicios y la mortalidad materna (15).

Los partos institucionales han aumentado considerablemente y casi la mitad de las mujeres ahora tienen sus partos atendidos por personal sanitario. La atención posnatal sigue siendo el área más descuidada y pocas mujeres son visitadas en estas semanas vulnerables después del parto. Estos datos ponen de relieve la evolución lenta, a pesar del programa Nacional de maternidad sin riesgo $(22,23)$.

\section{CONCLUSIONES}

Existe fuerte asociación entre factores socioeconómicos y los servicios de Salud. Los datos sobre servicios obstétricos y de salud reproductiva son difíciles de evaluar porque no se han estudiado en detalle los factores socios económicos involucrados, específicamente los que influyen en la decisión de la mujer de solicitar atención médica y los que demoran o evitan que la mujer reciba atención.

La relación entre las desigualdades de salud de la población y los factores sociales y económicos está siendo investigada por la Epidemiología. De manera general, las evidencias apuntan a un fuerte gradiente social en el ámbito de la salud de la población, invariablemente desfavorable para los grupos socialmente menos privilegiados. Las mujeres buscan el acceso a la atención de emergencias obstétricas, pero debido a una variedad de problemas, la atención apropiada a menudo se retrasa. El cuidado desorganizado de la salud con la falta de respuesta rápida a las emergencias, es un factor importante que contribuye a una alta tasa de mortalidad. Se destaca que la naturaleza de los problemas identificados y las intervenciones dirigidas a reducir la mortalidad materna y la morbilidad requiere una educación de la comunidad extensa. La educación y un sistema de salud eficaz y eficiente, especialmente durante el embarazo y el parto, están fuertemente relacionados con mortalidad materna. Además, los factores macro económicos están relacionados y podrían estar influyendo en los demás. Las mujeres con nivel socio económico bajo, a menudo son ignoradas, incluso si necesitan ayuda médica. El logro educativo, el nivel de empleo y la disponibilidad de las instalaciones sanitarias son indicadores de status socioeconómico de las mujeres. La pobreza es la mayor causa de mortalidad materna. Se requiere de intervenciones eficaces en salud pública basadas en una comprensión clara de percepciones de las mujeres sobre los servicios de atención materna dentro de su contexto cultural.

CONFLICTOS DE INTERÉS: ninguno que declarar.

FINANCIACIÓN: recursos propios de los autores.

\section{REFERENCIAS BIBLIOGRÁFICAS}

1. Organización Panamericana de la Salud. OMS. Mortalidad materna a nivel mundial. 1996.

2. Organización de las Naciones Unidas (ONU). Salud materna y neonatal: Situación actual. Disponible en: URL: http://issuu.com/dr.ppach/docs/salud_materna_y_neonatal_2009.

3. Bashour H, Asmaa A, Jabr A, Cheikha S, Tabbaa M, Lahham M, et al., Maternal mortality in Syria. Trop Med Int Health 2009; 1122 -1127.

4. Romero G, Espitia A, Ponce A, Huerta L. Risk factors of maternal death in Mexico. World Health Organization 2007; 34(1): 21-25.

5. Magadi M, IAN D, Nyovani Madise. Analysis of factors associated with Maternal Mortality in Kenyan Hospital. Journal of Biosocial Science 2001; 33(3), 375- 389.

6. Midhet $\mathrm{F}$, Becker $\mathrm{S}$, Berendes $\mathrm{H}$. Contextual determinants of maternal mortality in Rural Pakistan. Soc Sci Med 1998; 46 (12): 1587 - 1598.

7. Konteh R. Socio economic and other variables affecting maternal mortality in Sierra Leona. 
Community Development Journal. 1990; $49-64$.

8. Taguchi N, Kawabata M, Maekawa M, Maruo T, Aditiawarman and Dewata L. Influence of socioeconomic background and antenatal care programmes on maternal mortality in Surabaya Indonesia. Trop Med Int Health 2003; 8(9): 847-852.

9. Alvarez J, Gil R, Hernandez V, Gil A. Factors associated with maternal mortality in Subsaharan Africa. BMC Public Health 2009; 9- 462.

10. Chukuezi C. Socio Cultural Factors Associated with Maternal Mortality in Nigeria Social Science \& Medicine 2010; 1 (5): 22-26.

11. Hoj L, Silva D da, Hedegaard K, Sandstrom A. Factors associated with Maternal Mortality in Rural Guinea Bissau. An International Journal of Obstetric \& Gynaecology 2002; 109(7),792799.

12. Lubbock L A, Stephenson R. Utilization of maternal health care services in the Departament of Matagalpa, Nicaragua. Pan American Journal of Public Health 2008; 24(2), 75-84.

13. Souza J, Cecatti J, Faundes A, Morais S, Villar J, Carroli G, et al., Maternal near miss maternal death in the world health organizations. Bulletin of the World Heald Organization 2010; 88(2), 113-119.

14. J Li. C Luo, R Deng, Jacoby $\mathrm{P}, \mathrm{N}$ de Klerk. Maternal Mortality in Yunnan, China. An International Journal of Obstetrics \& Gynaecology 2007; 114(7), 865-874.

15. Ronsmans C, Etard J F, Walraven G, Hoj L, Dumont A, Bernis L de, et al. Maternal Mortality and access to obstetric services in West Africa. Tropical Medicine \& International Health 2003; 8(10), 940-948.

16. Walraven G, Telfer M, Rowley J, Ronsmans C. Maternal Mortality in rural Gambia. World Health Organization 2000; (5), 603.

17. Agudelo A, Belizan J. Maternal morbidity and mortality associated with interpregnancy interval: cross sectional study. British Medical Journal 2000; 1255-9.

18. Islam M, Chowdhury R, Chakraborty N, Bari W, Akhter H. Factors associated with delivery complications in rural Bangladesh. The European Journal of contraception and Reproductive Health Care 2004; 203-213.

19. Aggarwall A, A. P, B N, Bhattacharya. Risk factors for maternal mortality in Delhi slums. Indian Journal of Medical Sciences 2007; 61, 517.

20. Cham M, Sundby J, Vangen S. Maternal mortality in the rural Gambia a qualitative study on access to emergency obstetric care. Reproductive Health 2005; 2, 3-8.

21. Arps S. Threats to safe motherhood in Honduras Miskito Communities. Soc Sci Med 2009; 579 $-586$.

22. Kranti S, Mavalankar D, Ramani K, Upadhyaya M, Bharati S, et al., Maternal health situation in India. Journal of Health Population and Nutrition 2009; 27(2), 184- 201.

23. Okolocha Ch, Chiwuzie J, Braimoh S, Unuigbe J, Olumeko P. Socio cultural factors in maternal morbility and mortality. Journal Epidemiology Community Health. 1998; 293- 297.

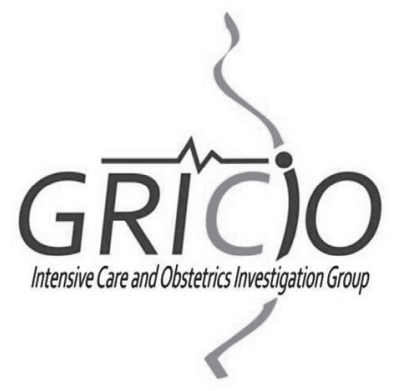

\section{GRUPO DE INVESTIGACIÓN: GRICIO}

LÍNEAS: Hemorragia obstétrica. Morbilidad materna extrema y mortalidad materna. Sepsias en el embarazo. Trastornos hipertensivos del embarazo. Ventilación mecánica en la embarazada Email: jocherojas2005@hotmail.com 